\title{
LA UNIVERSIDAD NACIONAL DE EDUCACIÓN A DISTANCIA (UNED) DE ESPAÑA
}

\author{
(THE NATIONAL UNIVERSITY OF DISTANCE EDUCATION (UNED) FROM SPAIN)
}

Lorenzo García Aretio

Universidad Nacional de Educación a Distancia (España)

\section{RESUMEN}

Tras una breve contextualización del entorno educativo español, en el artículo se presenta a una de las diez mayores universidades unimodales del mundo, la Universidad Nacional de Educación a Distancia (UNED) de España: su historia, estructura territorial, sus programas académicos, los docentes, los estudiantes, la metodología, los materiales, las unidades de apoyo a la docencia e investigación, la evaluación, etc. Hoy cuenta con casi 180.000 estudiantes. En sus 35 años de vida ha venido siendo pionera en la aplicación de una metodología que hoy se extiende por todo el mundo como forma de hacer accesible la educación y la cultura a todos a lo largo de toda la vida.

Palabras Clave: enseñanza superior, educación a distancia, tecnologías, UNED.

\begin{abstract}
UNED is one of the greatest unimodal universities of the world. Their history, territorial structure, academic programs, teachers, students, methodology, materials, supporting units for teaching and research, the evaluation, etc. Today have around 180. 000 students. In their 35 years of life it has been pioneer in the application of a methodology that today extends all over the world as a way of doing education and culture accessible for all.
\end{abstract}

Key words: higher education, distance learning, technologies, UNED. 
La educación a distancia en España se consolida, como en otros muchos países, lentamente a lo largo del pasado siglo XX. En 1903, surge la primera experiencia de enseñanza por correspondencia, protagonizada por Julio Cervera Baviera que crea las Escuelas Libres de Ingenieros. Años más tarde comenzaron algunos centros privados de enseñanza por correspondencia (García Aretio, 1999). Sin embargo, no será hasta 1960 cuando el gobierno autoriza el establecimiento de centros docentes y de estudios a distancia, encaminados a atender a la población diseminada en núcleos rurales de difícil acceso.

"En la década de los 60, la sociedad comienza a plantearse nuevas demandas educativas. Los fuertes movimientos migratorios y el despertar del mundo rural hacen aumentar extraordinariamente el número de estudiantes que no encuentran respuesta en el sistema tradicional; $y$ es en forma de 'alumnado libre' donde se puede encontrar el embrión de lo que luego sería la educación a distancia propiamente dicha. En aquella época los estudiantes libres llegan a alcanzar un tercio del total del alumnado" (MEC, 1995, p. 11).

En 1962 se inicia un proyecto de Bachillerato radiofónico y un año después se crea el Centro Nacional de Enseñanza Media por Radio y Televisión, que se transforma en 1968 en Instituto Nacional de Enseñanza Media a Distancia (INEMAD). Desde el ámbito privado destaca la experiencia de Radio ECCA (Emisora Cultural Canaria) que emitió su primera clase radiofónica en 1965. ECCA viene utilizando desde entonces, con algunas variantes, el denominado sistema tridimensional que conjuga la interacción de tres elementos, los esquemas impresos, la clase radiofónica y la tutoría presencial y a distancia. Este sistema ha sido exportado con éxito a otros países, sobre todo del área latinoamericana (García Aretio, 1994).

Pero será ya en 1970, al promulgarse la Ley General de Educación (LGE), cuando oficialmente se prevé la implantación de la educación a distancia en los diferentes niveles educativos. En su artículo 47.1 establece que, a fin de ofrecer las oportunidades para proseguir estudios a quienes no pueden asistir regularmente a los centros ordinarios, el Ministerio de Educación y Ciencia reglamentará las modalidades de enseñanza por correspondencia, radio y televisión. Más adelante, en al artículo 90, se refiere a los centros que imparten exclusivamente enseñanza a distancia. Con estas premisas se crean poco después el Instituto Nacional de Bachillerato a Distancia (INBAD, 1975) y el Centro Nacional de Educación Básica a Distancia (CENEBAD, 1979), instituciones destinadas a atender la enseñanza básica. Para impartir la educación superior se constituyó en 1972 la Universidad Nacional 
de Educación a Distancia (UNED). De esta forma, se fomentó el progreso cultural y el desarrollo social del país al garantizar el derecho a la educación de todo ciudadano en cada uno de los niveles educativos (García Aretio, 1994).

A partir de esta ley, la idea de educación a distancia se fue afianzando, y todas estas iniciativas han ido consolidándose a lo largo de las últimas décadas del siglo XX. Ejemplo de ello es la creación en 1992 del Centro para la Innovación y Desarrollo de la Educación a Distancia (CIDEAD) que dio respuesta a la Ley de Ordenación General del Sistema Educativo (LOGSE) de 1990 y que considera a la enseñanza a distancia como una de las vías de respuesta a la organización de la educación de las personas adultas. Este Centro pretende la investigación sobre necesidades de formación, la planificación de la oferta educativa, la ordenación académica, la formación del profesorado, la elaboración, seguimiento y evaluación de los medios didácticos, el diseño y elaboración de instrumentos de evaluación de los estudiantes, la incorporación de las nuevas tecnologías y la atención educativa de los estudiantes en casos excepcionales (García Aretio, 1994; 2002).

En la actualidad la mayoría de las universidades presenciales imparten programas o cursos a distancia, fundamentalmente a través de los nuevos sistemas de tecnologías avanzadas. Esta realidad viene a ser refrendada en la Ley Orgánica de Universidades (LOU, 2001, reformada en 2007), en la que se reconoce de forma explícita que toda institución superior podrá impartir sus enseñanzas tanto en la modalidad presencial como no presencial. También debemos mencionar a la Universitat Oberta de Catalunya (UOC), creada en 1995, universidad no presencial que propone un nuevo modelo educativo en el marco de la enseñanza universitaria. La UOC basa su sistema educativo en la implantación de un Campus Virtual. En otro artículo de este monográfico se presenta tal universidad.

\section{LA CREACIÓN DE LA UNED}

La novedad que la Ley General de Educación (LGE) de 1970 aportó al ámbito universitario español supuso la apertura de las rigideces estructurales del sistema convencional. Por primera vez se considera la educación como una permanente tarea inacabada, planteando por ello entre sus objetivos básicos los de ofrecer a todos una igualdad de oportunidades para acceder a la formación universitaria tanto inicial como permanente (García Aretio, 1986; 2001). En este sentido, introduce la modalidad de enseñanza a distancia, como medio que facilita la concreción del derecho a la educación de todo ciudadano. 
Para dar cumplimiento a este principio se constituyó una Comisión Gestora (1971) a la que se encomendó la creación de una universidad con la metodología de la enseñanza a distancia, modelo que ya se venía experimentando con éxito en otros países. Se trataba de implantar una enseñanza impartida a distancia de forma exclusiva, mediante la utilización de aquellos medios técnicos que en cada momento resultasen más idóneos, de tal modo que la Universidad pudiese "extender su acción a sectores de población que en aquella época, por razones de índole diversa, carecían de oportunidades o tenían dificultades graves para cursar estudios universitarios" (LGE, 1970). Así, se consideró que se lograrían mejor determinados objetivos contemplados en la Ley, tales como el de fomentar el progreso cultural y el desarrollo social del país desde la perspectiva del principio de igualdad de oportunidades (García Aretio, 1986; 2002).

Esta Comisión Gestora tenía como objetivo realizar un amplio estudio que incluyese, entre otros, los aspectos académicos y financieros del proyecto, junto con un análisis comparado de lo realizado en otros países, con el fin de organizar la puesta en marcha de esta nueva institución.

Un año más tarde, mediante el Decreto de 18 de agosto de 1972 se creó la Universidad Nacional de Educación a Distancia (UNED), universidad de titularidad pública, que impartiría sus enseñanzas en todo el territorio nacional desde la Sede Central en Madrid (España) y a través de una red de Centros Asociados distribuidos por todo el país y en numerosos países extranjeros. Actualmente es la única institución de educación superior legalmente reconocida que imparte enseñanzas de todos los ciclos universitarios en toda España (LOU, 2001, reformada de 2007).

La UNED opta por una modalidad de enseñanza a distancia basada en el modelo único, unimodal, o de exclusiva impartición de docencia a distancia. Para ello se establece, asimismo, una organización técnica, metodológica y administrativa propia para el desarrollo de esta modalidad a distancia (García Aretio, 1995; 2001).

La recién nacida UNED dedica sus primeros años a aumentar el número de alumnos, que va creciendo en progresión geométrica. Es preciso entonces adecuar su estructura a las necesidades docentes. Se crean los dos primeros vicerrectorados, uno de Humanidades y otro de Ciencias, y se abre una oficina de atención al público. Las unidades didácticas se envían a los estudiantes a sus casas, por correo postal, totalmente gratis.

El siguiente paso es llevar la educación superior a los núcleos de población, alejados de las grandes metrópolis, que no disponen de universidad. La creación de 
centros regionales servirá para asentar la UNED y su peculiar método docente en toda la península y en las islas. En estos centros los tutores actuarán como guías y asesores de los alumnos.

\section{LA ESTRUCTURA CENTRAL Y PERIFÉRICA DE LA UNED}

La UNED, como las restantes Universidades públicas españolas, cuenta con autonomía estatutaria o de gobierno, autonomía académica o de planes de estudio, autonomía financiera o de gestión y administración de sus recursos y, finalmente, autonomía para seleccionar y promocionar al profesorado, dentro del respecto de los principios de mérito, capacidad y no discriminación que atañen a todo puesto de trabajo dependiente del Estado (García Aretio, 1995; 2001), todas ellas reguladas por la legislación universitaria actual (LOU, 2001, reformada en 2007).

Una singularidad específica de esta Universidad es la de contar con una red de Centros Asociados repartidos por toda la geografía española y en el extranjero, que resultan imprescindibles para aplicar este modelo eficazmente. Los Centros Asociados, constituidos en consorcios o fundaciones con personalidad jurídica propia, forman parte de la estructura académica de la UNED. Son, asimismo, entidades esenciales para el normal funcionamiento de la Universidad, al tener lugar en ellos las actividades fundamentales de sus estudiantes: matrícula, tutorías, exámenes, etc. Representan, por tanto, la imagen que la sociedad tiene de la Universidad.

En realidad, si importante es el papel que desempeñan desde el punto de vista administrativo, de mucha mayor relevancia es su función de apoyo al aprendizaje de los estudiantes. Los Centros Asociados ofrecen a los estudiantes la necesaria orientación tutorial para el estudio, la posibilidad de acceso a una biblioteca y a una mediateca, así como la asistencia y participación activa en las diferentes actividades culturales organizadas por ellos. Por otra parte, los Centros Asociados proporcionan a los estudiantes de esta Universidad el necesario ambiente académico y cultural, aconsejable para conseguir la más completa formación universitaria. Su objetivo esencial es facilitar la relación entre la Sede Central, ubicada en Madrid, donde trabajan los equipos docentes de cada asignatura, y los estudiantes. Esta tarea resulta esencial, no sólo a escala administrativa sino también académica y cultural.

Desde un punto de vista académico, los Centros Asociados dependen de la Universidad y de sus órganos de gobierno, y los Profesores tutores deben seguir las directrices marcadas por el correspondiente Departamento de la Sede Central. 
En la vertiente económico-administrativa dependen del respectivo Patronato, Consorcio o Fundación integrados, además de por la propia Universidad, por distintas instituciones y entidades de ámbito local, provincial o autonómico, que colaboran al sostenimiento económico de los Centros, y que constituyen el órgano de participación de la sociedad en la vida universitaria del Centro Asociado (García Aretio, 1996; 2002).

Entre las funciones que habitualmente desarrollan los Centros Asociados, podemos destacar las siguientes, señaladas en los Estatutos de 2005:

- Desarrollar las correspondientes actividades docentes e investigadoras en coordinación con los departamentos.

- Fomentar en su entorno el desarrollo de actividades científicas y culturales.

- Poner a disposición de sus miembros los medios y servicios adecuados para el desarrollo de sus fines.

- Facilitar las relaciones administrativas entre los estudiantes y la universidad.

- Contribuir a la organización de las pruebas presenciales, en colaboración con los correspondientes servicios de la universidad.

Para el mejor desarrollo de todas estas funciones, la UNED ha puesto recientemente (2006) en marcha el denominado Plan ATECA (Arquitectura de Tecnología Educativa para los Centros Asociados) que, entre otros objetivos, pretende el desarrollo de una herramienta docente Audio-Visual IP (AVIP) para la tutoría telemática a distancia (TTD) que integra tecnología síncrona, aprovechando los avances arquitectónicos de los sistemas informáticos de la UNED, con el fin de que la oferta docente de los Centros llegue a partes del territorio español donde hoy no lo hace, así como que se posibilite una utilización más amplia de las sesiones y materiales de apoyo generado por los propios Centros.

La UNED cuenta actualmente con 61 centros repartidos por toda la geografía española. Dichos centros están interconectados gracias, entre otros factores, a las nuevas tecnologías que permiten un alcance directo a cualquier punto del país.

Los estudiantes que residen fuera de España pueden acceder a la oferta educativa de la UNED a través de la Red de Centros en el extranjero que esta Universidad mantiene en los siguientes países: 
- Alemania (Berlín), Argentina (Buenos Aires), Bélgica (Bruselas), Brasil (Sao Paulo), Francia (París), México (México), Reino Unido (Londres), Suiza (Berna), Venezuela (Caracas) y Perú (Lima). Estos Centros se han creado y se mantienen en virtud del Convenio suscrito entre el antiguo Instituto Español de Emigración, hoy Dirección General de Emigración del Ministerio de Trabajo y Asuntos Sociales y la UNED.

- Guinea Ecuatorial: Centros de Malabo y Bata, que la UNED mantiene en colaboración con la Agencia Española de Cooperación Internacional (AECI).

En los Centros en el extranjero se celebran las pruebas presenciales y se realizan y tramitan las matrículas. El estudiante también recibirá a través de los Centros indicaciones para el mejor desarrollo de sus estudios, existiendo en la mayoría de ellos tutorías de orientación general sobre los estudios y carreras con mayor implantación.

Los Centros en el extranjero disponen de una biblioteca básica con las publicaciones editadas por la UNED y los libros recomendados para las diferentes asignaturas. La UNED ofrece también a sus estudiantes la posibilidad de examinarse en Roma, Nueva York, Munich y Colonia, aunque en estas ciudades no existen Centros.

Como resulta obvio, una figura clave para la docencia en los Centros Asociados es la del profesor-tutor, figura que se aborda en otro apartado de este trabajo.

\section{EL GOBIERNO DE LA UNED}

Los órganos de gobierno de la UNED son similares a los de las restantes Universidades presenciales públicas españolas. La composición, atribuciones y modo de elección vienen determinados por la normativa universitaria general (LOU, 2001, 2007) y por los Estatutos de la UNED (los anteriores de 1985 y los actuales de 2005), que se concretan en órganos colegiados y unipersonales para su gobierno y gestión.

Entre los órganos colegiados destaca el Claustro universitario -máximo órgano de representación de la comunidad universitaria- que preside el Rector y está integrado por representantes de toda la comunidad educativa (profesores funcionarios y contratados, tutores, personal de administración y servicios y 
estudiantes). Al claustro universitario le corresponde conocer y debatir la gestión de la universidad y las líneas generales de actuación en los distintos ámbitos de la vida universitaria.

Como en todas las Universidades de España existe un Consejo Social que es el órgano de participación de la sociedad en la universidad y de permanente colaboración entre ambas, y contribuye a la prestación del servicio público de la educación superior que compete a la UNED mediante el ejercicio de sus atribuciones y la promoción de las iniciativas conducentes a un efectivo apoyo financiero por parte de las organizaciones sociales.

El Consejo de Gobierno es, como en las restantes universidades españolas, el órgano colegiado de gobierno de la universidad al que corresponde establecer sus líneas estratégicas y programáticas, así como las directrices y procedimientos para su aplicación en los ámbitos de organización de las enseñanzas, investigación, recursos humanos y económicos y elaboración de los presupuestos.

Cada Facultad o Escuela Técnica son gobernadas por sus respectivas Juntas y presididas por los Decanos (Facultades) o Directores (Escuelas) correspondientes. Son los órganos de deliberación y decisión de estos Centros.

De acuerdo con la normativa española, estas Facultades o Escuelas están organizadas en Departamentos, de acuerdo a las diferentes áreas de conocimiento científico y técnico. Éstos son los órganos básicos encargados de organizar y desarrollar la investigación y las enseñanzas propias de su área (LOU, 2001; 2007).

\section{LOS PROGRAMAS ACADÉMICOS DE LA UNED}

\section{Los estudios oficiales o reglados}

Tanto los estudios que se imparten en esta universidad, como los títulos que otorga, son semejantes a los que se desarrollan en las universidades presenciales.

En lo referente al tipo de estudios, carreras y programas que imparte la UNED, hemos de considerar en primer lugar los estudios reglados, es decir, aquellos que llevan a la obtención del Título Universitario oficial de Diplomado, Licenciado o Doctor en la coyuntura actual pero que, de acuerdo con las propuestas del Espacio Europeo de Educación Superior, en breve pasarán a denominarse Grado, Máster y Doctor. En la siguiente tabla se puede apreciar una distribución de titulaciones 
oficiales por Facultades y Escuelas, con especificación del número de estudiantes que siguieron los estudios en ellas:

\begin{tabular}{|c|c|}
\hline FACULTADES Y TITULACIONES & $\begin{array}{l}\text { ESTUDIANTES } \\
\text { MATRICULADOS } \\
(2005 / 2006)^{1}\end{array}$ \\
\hline \multicolumn{2}{|c|}{ 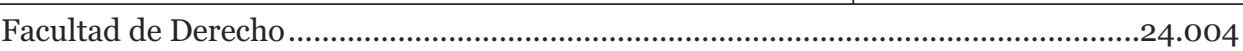 } \\
\hline Licenciatura en Derecho & 24.004 \\
\hline \multicolumn{2}{|c|}{ 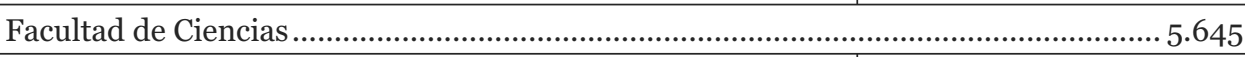 } \\
\hline Licenciatura en Físicas & 1.294 \\
\hline Licenciatura en Químicas & 1.372 \\
\hline Licenciatura en Matemáticas & 1.026 \\
\hline Licenciatura en Ciencias Ambientales & 1.953 \\
\hline \multicolumn{2}{|c|}{ Facultad de Ciencias Económicas y Empresariales ...................................................... 18.538 } \\
\hline Licenciatura en Admón. Dirección de Empresas & 9.487 \\
\hline Licenciatura en Economía & 3.332 \\
\hline Diplomatura en Turismo & 5.719 \\
\hline \multicolumn{2}{|c|}{ 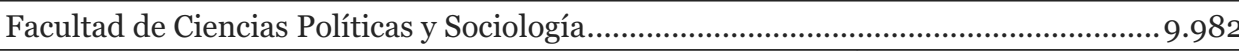 } \\
\hline Licenciatura en Ciencias Políticas & 3.279 \\
\hline Licenciatura en Sociología & 2.190 \\
\hline Diplomatura en Trabajo Social & 4.513 \\
\hline \multicolumn{2}{|c|}{ 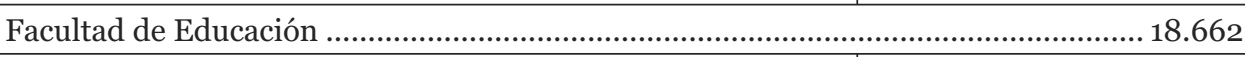 } \\
\hline Licenciatura en Pedagogía & 2.622 \\
\hline Licenciatura en Psicopedagogía & 6.328 \\
\hline Diplomatura en Educación Social & 9.712 \\
\hline \multicolumn{2}{|c|}{ 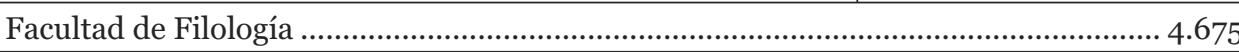 } \\
\hline Licenciatura en Filología Hispánica & 2.303 \\
\hline Licenciatura en Filología Inglesa & 2.372 \\
\hline \multicolumn{2}{|c|}{ 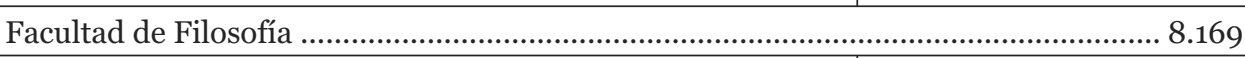 } \\
\hline Licenciatura Filosofía & 2.160 \\
\hline Licenciatura en Antropología Social y Cultural & 6.009 \\
\hline \multicolumn{2}{|c|}{ 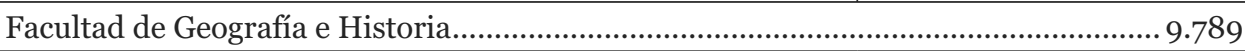 } \\
\hline Licenciatura en Historia & 9.789 \\
\hline \multicolumn{2}{|l|}{ Facultad de Psicología ................ } \\
\hline Licenciatura en Psicología & 17.560 \\
\hline \multicolumn{2}{|c|}{ 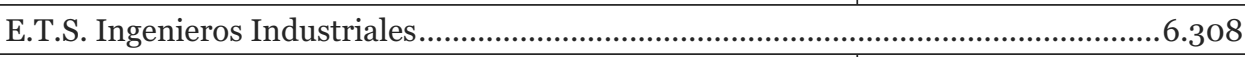 } \\
\hline Ingeniería Industrial & 3.384 \\
\hline
\end{tabular}




\begin{tabular}{|c|c|}
\hline Ingeniería Técnica Industrial Electrónica Industrial & 1.226 \\
\hline Ingeniería Técnica Industrial en Mecánica & 1.698 \\
\hline E.T.S. Ingeniería Informática.................................................................................12.439 \\
\hline Ingeniería Técnica en Informática de Sistemas & 6.355 \\
\hline Ingeniería Técnica en Informática de Gestión & 4.338 \\
\hline Ingeniería Informática & 1.746 \\
\hline TOTAL TITULACIONES OFICIALES....................................................135.771 \\
\hline
\end{tabular}

En los próximos cursos académicos esta oferta se va a ampliar considerablemente con otras carreras que irán dando respuesta a las necesidades sociales. En concreto, en el curso académico 2006-2007, además de las 26 titulaciones señaladas en la tabla, se han empezado a impartir dos carreras más, Ingeniero Técnico Industrial especialidad electricidad y Diplomado en Ciencias Empresariales. Por tanto, al día de hoy, pueden estudiarse en la UNED 28 carreras universitarias oficiales.

Respecto al Doctorado hemos de señalar que todas las Facultades y Escuelas Técnicas Superiores ofertan estudios de Doctorado, que se rigen por idéntica normativa que los restantes doctorados que se imparten en las universidades convencionales españolas. La obtención del Diploma de Estudios Avanzados (DEA) está organizada en dos años académicos, requisito imprescindible para poder acceder al desarrollo y defensa de la Tesis Doctoral.

Actualmente (curso académico 2006-2007) se están impartiendo 117 programas de doctorado diferentes, de ellos 19 tienen un carácter interuniversitario y 10 cuentan con la apreciada Mención de Calidad. Dentro de esos programas el número de cursos de doctorado del período de docencia en toda la UNED alcanza hoy un total de 1413 y los alumnos que realizan el trabajo de investigación, previos a la defensa del Diploma de Estudios Avanzados (DEA), son en la actualidad un total de 1050. El resto, hasta los 3554 que conforman el grupo total de alumnos de doctorado, se encuentran elaborando sus Tesis Doctorales.

Durante el curso académico 2005-2006 se defendieron un total de 137 Tesis Doctorales en las diferentes Facultades y Escuelas.

En consecuencia, en las enseñanzas regladas, Diplomatura, Licenciatura y Doctorado, durante el curso académico 2005-2006 la UNED contó con 135.771 + 3.554 un total de 139.325 estudiantes. 


\section{Los estudios no reglados o de formación continua}

Todas las Universidades a distancia del mundo ofrecen a la sociedad en la que se insertan, programas de formación permanente que atienden a las necesidades de formación de diferentes colectivos de profesionales (García Aretio, 1998). En la última década, la educación permanente o continua, de nivel superior, orientada tanto a la formación académica del postgrado como a la capacitación profesional ha alcanzado un extraordinario desarrollo en el sistema universitario español. Resultan evidentes las posibilidades que ofrece la metodología a distancia para atender las necesidades de formación de los profesionales en servicio.

Los Estatutos de la UNED de 2005 apuntan también como uno de sus objetivos destacados, el de "establecer y desarrollar programas de educación permanente, promoción cultural, perfeccionamiento y actualización profesional”. Este objetivo, en realidad, aparece desde la propia fundación de la UNED. Así, desde el principio esta Universidad abordó esa tarea y durante los últimos cursos académicos ofrece a la sociedad numerosos cursos diferentes que se articulan como enseñanzas no regladas o de Estudios de Postgrado, conducentes a la obtención de Títulos Propios y Diplomas de la UNED, que se desarrollan a través de los siguientes programas:

- Máster/Maestría (alto grado de especialización). Un mínimo de 50 créditos². Para acceder a estos estudios es requisito imprescindible poseer el título oficial de Licenciado, Ingeniero o Arquitecto.

- Especialista Universitario (especialización media). Un mínimo de 30 créditos que se imparten a lo largo de un curso académico. Para acceder a estos estudios también es requisito imprescindible poseer el título de Licenciado, Ingeniero o Arquitecto.

- Experto Universitario (especialización inferior). Un mínimo de 20 créditos que se imparten a lo largo de un curso académico. Para acceder a estos estudios es requisito imprescindible poseer el título de Diplomado, Ingeniero Técnico o Arquitecto Técnico, propios del Primer Ciclo Universitario.

- Programa de Formación del Profesorado. Son cursos destinados a graduados en cualquier ciclo universitario, especialmente a quienes ejercen la docencia en los distintos niveles educativos. Sus 12 créditos se cursan a lo largo de seis meses. 
- Programa de Enseñanza Abierta. Dirigido a posibilitar a cualquier persona la ampliación de sus conocimientos y el desarrollo y la promoción en algún campo del saber. Todos los cursos tienen 12 créditos. No existe requisito mínimo de acceso.

- Programa de Desarrollo Profesional. Atiende a una demanda de formación en respuesta a las necesidades e intereses del mundo del trabajo. Sus cursos están promovidos por entidades sociales y económicas que buscan el apoyo académico de la Universidad para la mejor calidad de sus acciones formativas. Se imparten a través de convenios con las entidades colaboradoras.

Se muestran seguidamente los datos correspondientes al curso académico 20052006 relativos al número de cursos y estudiantes matriculados en cada uno de los programas:

\begin{tabular}{|l|r|r|}
\hline Títulos Propios de Postgrado (2005-2006) & Estudiantes & \multicolumn{1}{|c|}{ Cursos } \\
\hline Experto Universitario & 3.422 & 82 \\
\hline Especialista Universitario & 971 & 29 \\
\hline Máster & 994 & 26 \\
\hline Formación del Profesorado & 3.700 & 201 \\
\hline Total Postgrado & 9.087 & 338 \\
\hline Otros Títulos Propios (2005-2006) & \multicolumn{2}{|c|}{186} \\
\hline Programa de Enseñanza Abierta & 4.606 & 38 \\
\hline Programa de Desarrollo Profesional & 1.454 & 24 \\
\hline Formación en el Área de la Salud & 1.605 & 248 \\
\hline Total Otros Títulos & 7.665 & 1 \\
\hline Otros Estudios (20o5-20o6) & & 10 \\
\hline Cursos Acceso Directo mayores de 25 años & 14.830 & 90 \\
\hline Centro Idiomas a Distancia (C.U.I.D.) & 2.889 & 125 \\
\hline Cursos de Verano & 2.616 & $\mathbf{7 1 1}$ \\
\hline Total Otros Estudios & 20.335 & $\mathbf{3 7 . 0 8 7}$ \\
\hline TOTAL GENERAL ESTUDIOS NO REGLADOS & & \\
\hline
\end{tabular}

Por tanto, fuera de los estudios reglados (oficiales) de la UNED, se atiende en programas de formación continua y otros cursos especiales a un total de 37.087, que sumados a los 135.771 de las enseñanzas regladas de diplomatura y licenciatura más 
los 3.554 de doctorado, hacen un total de 176.412 atendidos por la UNED durante el curso académico 2005-2006.

No se contabilizan en estos números a los participantes en los diferentes cursos y seminarios que se organizan desde los Centros Asociados de la UNED a lo largo de cada curso académico.

\section{EL PROFESORADO DE LA UNED}

\section{El profesorado de la Sede Central}

La UNED cuenta con dos tipos de profesorado, los de la Sede Central en Madrid y los de los Centros Asociados o profesores tutores. Los primeros son profesores que desarrollan las tareas docentes e investigadoras específicas de su área de conocimiento y materia, distribuidos por cuerpos y categorías idénticos a los de las restantes universidades españolas (Catedráticos, Titulares, Asociados, Ayudantes, Colaboradores, Contratados, Eméritos, etc.) (LOU, 2001; 2007). El acceso a las plazas de los diferentes tipos de profesores universitarios de la Sede Central exige los mismos requisitos que para ingresar en otras universidades públicas del Estado español.

En la UNED el profesor de la Sede Central en Madrid, no imparte clases tradicionales, sino que, atendiendo a la metodología de la educación a distancia, programa su materia, diseña y elabora los materiales didácticos adecuados a esa propuesta (impreso, audiovisual o multimedia). Asimismo a lo largo del curso atiende tanto a los profesores tutores de los Centros Asociados, dándoles las directrices adecuadas para el desarrollo de su tarea, como a los estudiantes a través de las consultas telefónicas, por correo, a través de la plataforma virtual..., la organización de videoconferencias, convivencias presenciales, programas de radio y televisión, etc.

Los datos relativos al profesorado de la Sede Central y correspondientes al curso académico 2005-2006, son los siguientes: 


\begin{tabular}{|c|c|c|c|c|c|}
\hline \multicolumn{6}{|c|}{ Personal Docente e Investigador en Facultades y Escuelas } \\
\hline & Hombres & $\%$ & Mujeres & $\%$ & Total \\
\hline \multicolumn{6}{|l|}{ Permanentes } \\
\hline Catedráticos & 136 & 82 & 29 & 18 & 165 \\
\hline Titulares Universidad & 259 & 47 & 297 & 53 & 556 \\
\hline Titulares Esc.Univ. & 46 & 50 & 46 & 50 & 92 \\
\hline \multicolumn{6}{|l|}{ No Permanentes } \\
\hline Asociados & 120 & 69 & 54 & 31 & 174 \\
\hline Ayudantes & 53 & 44 & 68 & 56 & 121 \\
\hline Ayudantes Esc. Univ. & 3 & 75 & 1 & 25 & 4 \\
\hline Ayudantes Doctor & 13 & 48 & 14 & 52 & 27 \\
\hline Colaborador (Doctor) & 38 & 49 & 39 & 51 & 77 \\
\hline Contradado Doctor & 18 & 31 & 40 & 69 & 58 \\
\hline Eméritos & 18 & 69 & 8 & 31 & 26 \\
\hline Personal Investigador & 38 & 51 & 36 & 49 & 74 \\
\hline Profesor Visitante & 1 & 50 & 1 & 50 & 2 \\
\hline Total & 743 & 54 & 633 & 46 & 1.376 \\
\hline
\end{tabular}

\section{El profesorado de los Centros Asociados (Profesores Tutores)}

La singularidad del tipo de docentes de la UNED la conforma el profesorado de los Centros Asociados, los profesores tutores. Hoy se cuenta con más de 6000 docentes tutores, cuya función esencial es la de orientar a los alumnos en sus estudios. La tarea fundamental de estos docentes de la UNED consiste en el desempeño de las tutorías presenciales a las que voluntariamente acuden los estudiantes matriculados en la materia correspondiente, con el fin de recibir las orientaciones oportunas para mejorar sus aprendizajes. Igualmente se tutela a los estudiantes a través de la plataforma virtual y, cada vez menos, a través del correo o teléfono.

En el marco de la función evaluadora, a los profesores tutores les corresponde la evaluación formativa, orientada a la comprobación del grado de logro de los objetivos durante el proceso de aprendizaje, la identificación de las lagunas, carencias y errores y la ayuda destinada a la superación de las mismas.

Según los Estatutos de la UNED de 2005, sus dos funciones esenciales son: 


\section{GaRCía \\ La Universidad Nacional de EduCaCión a Distancia (UNED) de España}

- Orientar al alumno en sus estudios, aclarar y explicar las cuestiones relativas al contenido de las materias cuya tutoría desempeñan, siguiendo las directrices del departamento.

- Informar al profesor responsable de cada asignatura del nivel de preparación de los estudiantes.

Los profesores tutores realizan la actividad tutorial presencial en los Centros Asociados y utilizan los medios tecnológicos de comunicación que la UNED adopta en su modelo educativo con el fin de propiciar tutorías telemáticas.

Para todo ello, los tutores deben seguir las directrices y el programa de enseñanza diseñado por el equipo docente de la asignatura en la Sede Central o Sede académica, con el material que éstos han elaborado, o escogido. Atienden a los estudiantes, ya sea presencialmente en la tutoría, o a distancia utilizando los medios que tienen a su disposición (teléfono, correo electrónico, página Web de la asignatura, plataforma virtual, correo postal, etc.). El tutor de la UNED ejerce funciones básicamente como las de motivador, facilitador y elemento de ayuda personal que está a disposición del estudiante a lo largo de todo el proceso de aprendizaje de la materia correspondiente. De esta manera, "el profesor - tutor deberá esforzarse en personalizar la educación a distancia mediante un apoyo organizado y sistemático, que posibilite el estímulo y orientación individual, la facilitación de las situaciones de aprendizaje y la ayuda para resolver las dificultades del material didáctico de apoyo al estudio independiente" (García Aretio, 1996, p.265).

En el curso académico 2005-2006, se contaba con los siguientes números relativos al profesorado de los Centros Asociados:

\begin{tabular}{|cccccc|}
\hline \multicolumn{5}{c}{ Profesorado Tutor en Centros Asociados de la UNED } \\
& Hombres & $\%$ & Mujeres & $\%$ & Total \\
Tutores & 4.209 & 67 & 2.044 & 33 & 6.253 \\
\hline
\end{tabular}

\section{LOS ESTUDIANTES DE LA UNED}

El tipo de estudiante de la UNED es, primordialmente, un alumno adulto. Su nivel de estudios es ligeramente superior a la media de los universitarios españoles, 
se trata en buen número de trabajadores a jornada completa, con responsabilidades familiares y que cursan estos estudios universitarios con el fin de mejorar sus expectativas profesionales, eligiendo la modalidad a distancia por razones prácticas (García Aretio, 1996).

Es de destacar que el sexo de los estudiantes de la UNED se ha ido equiparando progresivamente, hasta llegar en la actualidad (curso 2005-2006) a contar con un $51 \%$ de hombres y un $49 \%$ de mujeres.

Algunos datos destacados del estudio realizado en el curso 2002-2003 por Sánchez Elvira y otros, son los siguientes:

- En torno a un $60 \%$ de los estudiantes son solteros y un 30\% tiene uno o más hijos.

- Los hogares donde viven los estudiantes de la UNED, en un $74 \%$ están formados por entre 2 y 4 personas. Un $9 \%$ vive solo.

- La UNED cuenta entre sus estudiantes con un grupo que disfruta ya de alguna titulación universitaria (18\%); otro grupo importante lo conforman aquellos que han trasladado su expediente desde otra Universidad española (16\%). Los restantes acceden por vías ordinarias similares a los estudiantes de universidades presenciales.

- Un 76\% de los estudiantes encuestados en el curso 2002-2003, realizaban una actividad laboral remunerada.

De otro estudio anterior (García Aretio, 1999), entresacamos algunos datos que pueden resultar de interés:

- La mayoría de los estudiantes (76\%) no interrumpió nunca sus estudios desde que llegó a la UNED; tiende a matricularse del mismo número de asignaturas que el año anterior o de una más. El $35 \%$ de los estudiantes vienen matriculándose en cinco o más materias diferentes cada año académico.

- El 52\% de los estudiantes aprueba o supera al final de cada año sólo una o dos materias de entre las matriculadas. El 13\% de ellos supera cinco o más. 
- A cada una de las materias en que se matricula cada estudiante, el $47 \%$ de ellos les dedican entre una y tres horas semanales a su estudio. Cuatro o más horas semanales de estudio a cada materia o asignatura, le dedican el $31 \%$ de los estudiantes.

- Entre el 30 y el 40\% de los estudiantes (según carreras y Centros) asisten con frecuencia semanal a los Centros Asociados de la UNED para participar en las tutorías presenciales. Existe una moderada relación positiva entre frecuencia en la asistencia al Centro Asociado y éxito académico del estudiante.

- Respecto a las edades actuales de nuestro alumnado, valgan los datos que se muestran en la siguiente tabla.

Estudiantes matriculados según titulaciones y edad. Porcentajes verticales (Curso 2005-2006)

\begin{tabular}{|l|c|c|c|c|c|}
\hline & $(\mathbf{2 9 )} \%$ & $\mathbf{( 3 0 - 3 9 )} \%$ & $\mathbf{( 4 0 - 4 9 )} \%$ & (50 y más) \% & Media \\
\hline Curso de Acceso & 11,15 & 10,88 & 8,74 & 7,02 & 34,09 \\
\hline Diplomaturas & 27,08 & 21,43 & 16,38 & 7,86 & 33,25 \\
\hline Segundo ciclo & 12,28 & 8,71 & 9,18 & 8,04 & 35,42 \\
\hline Licenciaturas & 49,48 & 58,98 & 65,70 & 77,09 & 37,32 \\
\hline TOTAL & 100 & 100 & 100 & 100 & 35,96 \\
\hline
\end{tabular}

Son titulaciones con un marcado perfil masculino entre sus alumnos, las de Aministración y Dirección de Empresas, Economía, Filosofía, Ciencias Físicas, Matemáticas, Ciencias Químicas, Historia y las Ingenierías (Industrial e Informáticas). Las titulaciones donde prima el componente femenino son: Ciencias de la Educación/Pedagogía, Psicopedagogía, Filología Española/Hispánica, Filología Inglesa, Sociología y Ciencias Políticas.

Los planes de estudio a extinguir de diversas titulaciones son, como cabía esperar, los de las que tienen una estructura de edad más avanzada de sus alumnos. Dejando esta particularidad de los planes antiguos, las titulaciones menos jóvenes son: Filosofía, casi la tercera parte está formada por mayores de 45 años, y Ciencias Físicas, Matemáticas, Ciencias Políticas y Sociología con alrededor de la cuarta parte de los alumnos por encima de los 45 años. Debido a la edad de sus alumnos, parecen carreras de segunda oportunidad: tal vez para relanzar la vida laboral; tal vez, directamente, para relanzar la vida (Anuario UNED 2005-2006). 
En cuanto al acceso a las enseñanzas denominadas regladas u oficiales los requisitos son idénticos a los exigidos a aquellos estudiantes que cursan sus estudios en otra Universidad española. En las enseñanzas no regladas o no formales, en el caso de los postgrados, también el grado de exigencia en cuanto a la titulación previa para el acceso a los estudios, es el mismo que exigen las demás instituciones superiores.

\section{Atención a otras necesidades}

Desde siempre, la UNED ha tenido una vocación clara de cumplir una importante función social, al hacer llegar la educación superior a zonas geográficas y sectores sociales que con anterioridad se veían privados de esta posibilidad. La legislación española permite iniciar estudios universitarios a aquellos ciudadanos de 250 más años que, sin necesidad de haber superado los niveles oficiales requeridos de enseñanza preuniversitaria superen una prueba previa en la Universidad en la que pretenden estudiar.

Pues bien, prácticamente desde sus inicios, la UNED diseñó un Curso de Acceso Directo $(C A D)$ para mayores de 25 años con el fin de preparar adecuadamente a los estudiantes para la realización de la citada prueba de acceso. Son ya numerosos los licenciados de la UNED que hoy ocupan puestos relevantes en la sociedad española, en el mundo de la empresa, la cultura, la política, la educación, la economía, etc., que, sin disponer de estudios previos para el acceso a la Universidad, siguieron en la UNED el $C A D$ y los posteriores estudios universitarios que les permitieron una promoción social, económica y, sin duda, personal (García Aretio, 1985). Como ya se ha indicado, durante el curso académico 2005-2006, un total de 14.830 estudiantes siguieron este curso.

La UNED se viene destacando históricamente por realizar un notable esfuerzo en la atención académica de sujetos discapacitados físicos que, gracias a convenios suscritos por esta Universidad y otras organizaciones sociales y empresas están posibilitando el estudio universitario a un importante colectivo de minusválidos físicos, que de otra forma verían limitado su acceso a este nivel de estudio. Para ello existe una Unidad de Discapacidad que es un servicio dependiente del Vicerrectorado de Estudiantes y Desarrollo Profesional, cuyo objetivo principal es que los alumnos con discapacidad que deseen cursar estudios en esta Universidad, puedan gozar de las mismas oportunidades que el resto de estudiantes de la UNED. Con este fin, la Unidad coordina y desarrolla una serie de acciones orientadas a la asistencia, apoyo y asesoramiento que les permita, en la medida de lo posible, un desenvolvimiento 
pleno en el ámbito de la vida universitaria. Durante el curso académico 2005-2006 formaron parte del alumnado de la UNED un total de 3.430 discapacitados.

Otra destacada atención que la UNED viene prestando a la sociedad, prácticamente desde su creación, la conforma el programa de estudios universitarios en Centros Penitenciarios. Este programa es fruto del desarrollo de los Convenios firmados entre la Secretaría de Estado de Universidades e Investigación y la UNED, con otros organismos del Estado. Su objetivo es incrementar el nivel formativo y cultural de la población reclusa española en territorio nacional y establecimientos penitenciarios extranjeros, posibilitando, a través de la enseñanza abierta y a distancia, su acceso a los estudios universitarios en idénticas condiciones que el resto de los ciudadanos. Para ello, la UNED pone a disposición de los estudiantes internos en centros penitenciarios los siguientes servicios y materiales: sistemas de orientación, información y matriculación; material didáctico básico de las carreras universitarias y del Curso de Acceso Directo; tutorías semanales en los centros penitenciarios prioritarios de algunas asignaturas del Curso de Acceso y de las que se determinen en las principales carreras y apoyo del Centro Asociado de la UNED más próximo para los estudiantes en régimen abierto y en libertad condicional. Durante el curso académico 2005-2006 se atendió a una población reclusa de 801 estudiantes.

Ya se hizo mención más arriba a la labor que también se realiza con los alumnos que no residen dentro de la geografía española pero que pueden seguir sus estudios en la UNED a través de la red de centros en el extranjero. Durante el curso académico 2005-2006, siguieron estos estudios, fuera de España un total de 1.849 alumnos.

En la siguiente tabla mostramos resumidamente los datos del presente epígrafe.

\begin{tabular}{|c|r|r|r|r|r|}
\cline { 2 - 6 } \multicolumn{1}{c|}{} & Hombres & \% & Mujeres & \% & Total \\
\hline Con necesidades especiales & & & & & \\
\hline Discapacidad & 1.928 & 56,2 & 1.502 & 43,8 & 3.430 \\
\hline C. Penitenciarios & 703 & 87,8 & 98 & 12,2 & 801 \\
\hline En el Extranjero & 946 & 51,2 & 903 & 48,8 & 1.849 \\
\hline
\end{tabular}

\section{El Centro de Orientación, Información y Empleo (COIE)}

El COIE es un servicio especializado en orientación académica y profesional por el que los estudiantes y titulados de la UNED reciben ayuda a lo largo de sus estudios, 
desarrollo profesional y posterior inserción laboral. Este servicio gratuito permite a las empresas que colaboran con el COIE recibir candidatos con un perfil competitivo para cubrir tanto sus necesidades laborales como de prácticas.

Por tanto, el COIE ofrece ayuda personalizada durante los estudios con el fin de que los estudiantes:

- Aprendan a rentabilizar mejor los recursos que la UNED pone a su alcance,

- Utilicen ciertas técnicas de estudio autorregulado,

- Gestionen su tiempo de estudio,

- Afronten mejor los exámenes y

- Superen las dificultades de aprendizaje en el sistema a distancia.

A través del COIE el estudiante de la UNED tiene acceso a información y recursos adicionales para la formación: becas, cursos complementarios, oportunidades de estudiar en el extranjero o de realizar prácticas de trabajo en empresas, etc.

Y una vez que los alumnos finalizan sus estudios el COIE ofrece ayuda personalizada para planificar la búsqueda de empleo. Se dispone, igualmente de una bolsa de trabajo de alumnos y titulados de la UNED, a partir de la cual se preseleccionan candidatos de acuerdo con las ofertas de empleo o de prácticas recibidas por parte de la empresas. Y, finalmente, se informa a los estudiantes y graduados de las posibilidades de proseguir la formación y acceder a la información sobre una amplísima oferta formativa de postgrado y especializada existente en España y en el extranjero.

\section{LA METODOLOGÍA EN LA UNED}

La Ley General de Educación (LGE) de 1970, como se ha señalado más arriba, recogía dentro del apartado de Modalidades de enseñanza, que podría impartirse enseñanza universitaria por correspondencia y a través de la radio y la televisión. No cabe duda de que ese artículo de la LGE, escrito hace casi cuarenta años, era innovador en el ámbito educativo. Allí se contemplaba la posibilidad de seguir estudios universitarios utilizando tecnologías innovadoras aplicadas a la enseñanza/ aprendizaje.

Consecuentemente, como se recordará, el Decreto de creación de la UNED de 1972 dispuso que esta Universidad impartirá sus enseñanzas a través de la radio, 
la televisión, las cintas magnéticas y videomagnéticas y cualquier otro medio análogo. Lógicamente, en los Estatutos de la UNED de 1985 se indicaba asimismo que la modalidad de educación a distancia supone la aplicación de una metodología didáctica específica en donde se integran sistemas de comunicación y recursos que incluyen la utilización de medios impresos, audiovisuales y de las nuevas tecnologías, consideraciones que se reiteran en los Estatutos de 2005.

Cada asignatura se apoya tanto en los materiales impresos, audiovisuales... como en los telemáticos. Es decir, cada una cuenta con su correspondiente virtualización en la plataforma de la Universidad, en la que se incluyen tanto contenidos, orientaciones, enlaces... sobre la asignatura, como foros, chats... además de los medios tradicionales de la educación a distancia.

Cada asignatura emite también programas de radio, que se graban en los propios estudios de la UNED, así como programas de televisión educativa que se emiten todos los fines de semana a los que después aludiremos.

El modelo educativo de la UNED está basado en la posibilidad efectiva del estudio autónomo del alumno, siempre que se le facilite una adecuada organización de los contenidos con unos materiales y medios tecnológicos adecuados para el logro de su aprendizaje. En esta línea, se ha diseñado la enseñanza a distancia como un diálogo didáctico mediado entre el profesor y el estudiante que, separado físicamente de aquel, aprende de forma independiente y flexible pero aprovechando las posibilidades de las tecnologías colaborativas (García Aretio, 2001). La comunicación bidireccional entre profesor y estudiante no se pierde, al estar fundamentada en una planificación tecnológica de todo el proceso en el que están claramente programados cada uno de los recursos, elementos esenciales del aprendizaje guiado. Desde los materiales impresos, los recursos telemáticos, la comunicación síncrona y asíncrona... todo está entrelazado y diseñado para ayudar a cada estudiante al logro del éxito.

De acuerdo con el principio anterior, cada equipo docente prepara el material didáctico de su asignatura y selecciona los medios a utilizar para su transmisión, ateniéndose a las normas generales que al respecto se fijan. La decisión adoptada queda reflejada en el plan docente anual del Departamento correspondiente, de acuerdo con lo establecido en la normativa de la UNED. El equipo docente de cada asignatura tiene amplia libertad para decidir la combinación de medios que considere más adecuada para el estudio de su materia, respetando en todo caso las directrices establecidas al respecto por la Comisión de Metodología de la Universidad. Para la elaboración, así como para su edición, se cuenta con equipos de especialistas que 
asesoran y desarrollan toda la infraestructura necesaria y que están encuadrados en diferentes unidades e institutos que posteriormente se mencionarán.

En este proceso de aprendizaje cada estudiante, como decimos, cuenta desde el comienzo del curso con una planificación precisa de cada asignatura, y para su superación se le ofrecen la interacción de los siguientes medios: Medios impresos, Medios audiovisuales y Medios telemáticos. Se describen brevemente a continuación.

\section{El material impreso}

La UNED edita una amplia gama de publicaciones impresas destinadas a su alumnado: la Guía de carrera, una por titulación universitaria, que incluye las indicaciones académicas y administrativas básicas para el estudiante de cada carrera; las Unidades Didácticas o textos básicos de estudio, complementadas con Addendas y Guías Didácticas, destinadas a orientar al alumno en cuanto a métodos de estudio y contenidos de cada materia.

El texto, aun en la era informática y las telecomunicaciones, continúa siendo en la mayoría de las Universidades a distancia un elemento fundamental y básico que progresivamente se va complementando con otros recursos audiovisuales e informáticos. La UNED es hoy, de hecho, la mayor editorial universitaria española en volumen y diversidad de producción: desde los libros de todo tipo (materiales didácticos, libros de colecciones, revistas, etc.), hasta las publicaciones efímeras o de renovación anual (Guías Institucionales y de Carrera, de gran tirada, Programas de asignaturas, etc.).

El material didáctico en forma de libro impreso, habitualmente denominado Unidades Didácticas, constituye el instrumento fundamental del estudio en la UNED. Como norma general, buena parte de las asignaturas cuentan con sus propias Unidades Didácticas, elaboradas de acuerdo con las exigencias de la educación a distancia y capaces de permitir al alumno un estudio independiente.

Las Unidades Didácticas no se ciñen formalmente a un único esquema didáctico, pudiendo el equipo docente elegir el modelo que desea aplicar. No obstante, su elaboración tiene en cuenta las consideraciones generales propias de este tipo de materiales (García Aretio, 1997).

Antes de su publicación, el Instituto Universitario de Educación a Distancia de la UNED comprueba que las Unidades Didácticas nuevas o renovadas se adaptan a 
las exigencias de la educación a distancia y orienta a los docentes para su adecuada elaboración. La Comisión de Medios puede aprobar la sustitución de unas Unidades Didácticas por otro material impreso, a propuesta razonada del equipo docente y avalada por el Departamento correspondiente. En tal caso, es necesaria la confección de una Guía Didáctica para el estudio de la materia, suficientemente amplia y orientativa y que adecue el material propuesto para su estudio a distancia.

Las Unidades Didácticas suelen ser completadas por cualquier otro material escrito, audiovisual o informático que el equipo docente considere necesario, cuidando especialmente su disponibilidad y accesibilidad por parte de los estudiantes.

\section{Medios didácticos audiovisuales e informáticos}

Los medios audiovisuales ofrecen una variada selección de formatos que permiten atender a los distintos objetivos didácticos de cada asignatura. Así, cada medio ofrece unas posibilidades específicas. Como criterio general, la radio, la televisión, la videoconferencia y la plataforma virtual se utilizan para facilitar periódicamente una relación docente más directa entre profesores y estudiantes.

Los medios complementarios tecnológicos que utiliza la UNED como apoyo a la enseñanza, han existido desde el principio integrándose en su modelo singular y han evolucionado al ritmo de los avances tecnológicos. Así se han utilizado progresivamente las emisiones de radio, los programas de televisión, los vídeos educativos, los multimedia, la red de videoconferencia y, finalmente, Internet.

Ya en 1974 se creó la Dirección Técnica de la UNED que asumió la dirección conjunta de los correspondientes servicios, especialmente los medios audiovisuales y soportes de difusión utilizados en las actividades docentes. Su acción inicial se centró sobre todo en la programación radiofónica diaria que desde entonces ha venido ofreciendo la UNED a través de Radio Nacional de España. Desde aquel momento, todos los programas de radio producidos en la UNED, eran grabados y enviados a los Centros Asociados. Desde 1998 se viene ofreciendo a través de Internet toda esta programación radiofónica de la UNED. Hasta ese año 98, una vez emitida por Radio Nacional de España (RNE), esta programación era de complicado acceso para algunos y de imposible audición para otros. Por ello la UNED, consciente del valor que sus programas poseen para sus estudiantes y para un determinado público interesado en conocer aspectos científicos, culturales y sociales de actualidad, consideró oportuno difundirlos de manera más amplia a través de Internet. 
Desde el Curso 1991/92 la UNED viene utilizando también la televisión, actualmente los fines de semana desde La 2 de Televisión Española. Igualmente, los vídeos que se producen en la UNED, VHS antes y DVD ahora, abordan determinados temas de las diferentes materias. En otros casos conforman colecciones que curso tras curso se van ampliando. A finales del siglo pasado se inició el proyecto TeleUNED. El objetivo de este nuevo sitio Web en la página principal de la UNED, es triple:

- Difundir en directo los actos académicos y culturales que la propia Universidad organiza;

- Posibilitar el acceso online a su extensa videoteca organizada en torno a un amplio espectro de temas de interés científico y cultural y,

- Mostrar en diferido por Internet la programación de su televisión educativa.

Desde 1996, un servicio de telefonía avanzada posibilita, entre otras cosas, la comunicación y la transferencia de datos entre ordenadores y el servicio de respuesta telefónica automática que permite acceder a la información deseada que se encuentra almacenada en un ordenador en forma de voz.

Iniciada en 1993 la explotación de la Red Digital de Servicios Integrados (RDSI) por Telefónica de España, la UNED comenzó sus experiencias de videoconferencia de sala en octubre de ese año. Conforme la RDSI se fue extendiendo, la UNED fue llegando a todos sus Centros Asociados, constituyendo en su momento la más potente red de videoconferencia, para estos fines, de toda Europa.

La tradicionalmente denominada enseñanza asistida por ordenador no contó en la UNED con demasiadas experiencias. Determinadas materias de los programas de enseñanzas no regladas y algunas asignaturas, en su apartado de evaluación de aprendizajes, experimentaron entonces esta tecnología, como incursión en el multimedia.

La UNED dispuso de un Centro Servidor de Videotex desde 1990. Mediante este servicio los estudiantes conectados tenían acceso por ordenador, a determinadas informaciones de interés relacionadas con aspectos académicos e informativos generales de la Universidad. Existía un servicio de acceso público con información general de la Universidad, calificaciones de selectividad, preinscripciones, información sobre becas, cursos, ayudas y ofertas de empleo. Por cada Facultad y Escuela existía un servidor de acceso restringido, sólo para los estudiantes previamente dados de alta en él. Los estudiantes podían acceder a informaciones concretas de aquellas materias en las que estuviesen matriculados, calificaciones de 
las pruebas presenciales, comunicación con el profesor a través del correo electrónico $\mathrm{X} 400$, de entonces, etc.

Paralelamente a las experiencias con videotex se iniciaron otras propuestas de interacción profesor-estudiantes a través del ordenador. Una forma muy clásica fue la de establecer conexión entre un ordenador con modem y otro central al que podían acceder muchas personas para responder o preguntar u opinar. Se trataba del $B B S$ (Bulletin Board System). En la UNED el sistema BBS se utilizó entre 1994 y 97 en algunos cursos de postgrado con aceptables resultados.

La irrupción de Internet, afortunadamente, obligó a la progresiva supresión de los servicios de videotext y de $B B S$, dados su lentitud, costes elevados de conexión telefónica y limitadas prestaciones comparadas con las que se podían implementar a través de Internet.

Desde el curso académico 1995/96 se vienen realizando diferentes experiencias en la UNED que tienen como protagonista a Internet. Determinados cursos, fundamentalmente del ámbito de las enseñanzas no regladas, fueron progresivamente introduciendo en su metodología instancias formativas basadas en la comunicación a través de la red. A partir de ese año 1995 fueron muchos los profesores que progresivamente iban incorporando a su docencia el uso de un sitio Web, la utilización del correo electrónico y, posteriormente, listas de distribución y foros.

Desde el curso académico 1997/98 todos los profesores de la Sede Central de la UNED y todos los servicios administrativos de la Universidad dispusieron de ordenadores conectados a Internet y a redes locales. Todo el personal docente de la UNED desde ese mismo curso, contó desde sus domicilios con un acceso remoto a la Red UNED.

La consolidación de las diferentes propuestas, relativas a la enseñanza por Internet, tuvo su punto culminante en 1999, año en el que se inició un fuerte proceso de adaptación institucional al mundo de Internet. Se entendió que era importante que la UNED apareciera ante sus estudiantes y profesores con una única plataforma virtual de gestión del conocimiento porque eso supondría facilitar a los estudiantes el uso y a los profesores el enorme trabajo que supone producir un curso basado en la Web.

Se abordó el denominado Plan de Virtualización de la UNED que preveía la incorporación sistemática de servicios docentes en línea para las titulaciones que estaban actualizando sus planes de estudio. La virtualización se inició en el curso 
2000-2001 con los nuevos planes de estudio, iniciándose por el primer curso de cada una de las carreras. Desde 2005, prácticamente todos los cursos de todas las carreras regladas están virtualizados. Igualmente sucede con la mayoría de los estudios de postgrado y de educación permanente.

Después de numerosos análisis se optó por la plataforma que en ese momento estaba siendo más utilizada en el mercado de la enseñanza a distancia, la Web Course Tool (WebCT), herramienta para hacer cursos Web, dela British Columbia University . Esta plataforma, como el resto de los accesos restringidos de la UNED, está integrada dentro del ámbito de CiberUNED, centro de identificación de usuarios que personaliza la navegación según cada caso. Al mismo tiempo, desde el rectorado se impulsó el desarrollo de una plataforma basada en código abierto. Esta plataforma denominada $a L F$ se viene utilizando progresivamente en diferentes cursos de postgrado. Pero $a L F$ viene ofreciendo además un excelente servicio a multitud de comunidades de aprendizaje y grupos de investigación que la utilizan como herramienta de trabajo colaborativo. Hoy día, las dos plataformas conviven y las dos están conectadas con las aplicaciones de gestión académica con lo que se posibilita que los usuarios con un solo identificador puedan acceder a espacios de docencia y trabajo en grupo en cualquiera de las dos plataformas. El Plan de Virtualización de la UNED supuso la creación de una unidad de apoyo al profesorado para la transformación y producción de materiales orientados a los cursos virtuales.

Estas herramientas a través de los sistemas digitales se fueron utilizando en la UNED como valioso apoyo que se sumaba al conjunto de medios que ya integraban el sistema de enseñanza a distancia de la Universidad. Ha de aclararse que la utilización de la tecnología Internet, salvo en determinadas materias, tiene un carácter de uso voluntario para los estudiantes.

El profesorado durante estos años ha tenido la oportunidad de formarse en el uso de las tecnologías. Desde 1995, desde el Instituto Universitario de Educación a Distancia de la UNED se estableció un vasto plan de formación en tecnologías, de los docentes, tanto de la Sede Central como de los Centros Asociados. Esos planes de formación han posibilitado no sólo el uso de tecnologías por parte del profesorado, sino el entusiasmo de muchos equipos docentes convencidos de las inmensas ventajas que para el aprendizaje de calidad de los estudiantes comportan los sistemas digitales de enseñanza y aprendizaje.

En el momento actual, los centenares de asignaturas ya virtualizadas que conforman los planes de estudio de las diferentes titulaciones oficiales de la UNED, 
cuentan con una unidad o curso WebCT. En cada uno de estos cursos están inscritos, además de todos los estudiantes matriculados en esa asignatura, el equipo docente de la Sede Central y todos los tutores de los Centros Asociados que tutelan dicha materia. Este curso WebCT recoge toda la información necesaria para un seguimiento exitoso de la disciplina de estudio. El estudiante puede acceder a la guía de la carrera, a la guía didáctica, a materiales que complementan los impresos ordinarios, a sugerencias de documentos y artículos soportados en Internet, a clips multimedia producidos por la Universidad, a pruebas de evaluación, a exámenes de cursos anteriores, etc. Dado el gran número de estudiantes con que pueden contar ciertas asignaturas (bastantes de ellas cuentan con más de 4.000 estudiantes), el sistema de comunicaciones que se establecen en cada curso WebCT se estructura fundamentalmente a través de la herramienta "Foros", de la siguiente manera:

- Tablón de anuncios. En el mismo se deposita toda la información que el equipo docente de la Sede Central desea ir transmitiendo gradualmente a los estudiantes.

- Foro de Tutores. Este espacio de intercambio es de acceso restringido al equipo docente y los tutores. Es el vehículo ideal para la comunicación de todos los docentes que intervienen en cada asignatura, posibilitando una adecuada coordinación de la acción tutorial.

- Foro de estudiantes. Este foro sería como el espacio físico de la cafetería de las instituciones presenciales. Es un foro no moderado que facilita la comunicación entre pares. Es utilizado por los estudiantes para intercambiar opiniones sobre la asignatura, apuntes, resúmenes, exámenes de cursos anteriores, resolución de dudas entre ellos, etc.

- Foros de Centros Asociados. Cada Centro Asociado (cada profesor-tutor) cuenta con su propio foro. Es el lugar adecuado para la acción docente directa, para la comunicación del tutor con sus estudiantes. Cada uno de estos profesores-tutores es libre para organizar este espacio de acuerdo con sus preferencias, concordantes, en todo caso, con las directrices emanadas desde la Sede Central. Desde este foro cada tutor puede difundir la programación de la actividad tutorial en el Centro Asociado, resolver dudas de los estudiantes, distribuir, mediante ficheros adjuntos, materiales de apoyo, etc. Igualmente es un espacio ideal para debatir temas de interés relacionados con la materia.

- Foro de Consultas generales sobre la asignatura.

- Foro Guardia virtual.

- Etc. 
Naturalmente esta plataforma, como otras, permite que esta estructura pueda ser complementada con otros espacios de debate, de acuerdo con el número de estudiantes de la materia, la especificidad de ésta, etc. Así, se vienen utilizando en estos cursos de WebCT estas otras herramientas: calendario (ideal para transmitir todo tipo de avisos sujetos a una fecha), evaluación (para proponer ejercicios de autoevaluación o pruebas de evaluación a distancia), posibilidad de crear grupos de trabajo, además de las ordinarias posibilidades comunicativas de correo electrónico propio y salas de chat.

\section{APOYOS AL PROFESORADO}

De acuerdo con todo lo señalado en el punto anterior, es de destacar que la UNED a lo largo de su historia se ha venido dotando de diferentes unidades de apoyo a la docencia y a la investigación. Destacamos entre otros:

\section{Instituto Universitario de Educación a Distancia (IUED)}

$\mathrm{Al}$ nacer la UNED, se la dotó de un Instituto de Ciencias de la Educación (ICE) de características similares a los de las restantes universidades públicas, aunque sus funciones fueron generalmente diferentes a las propias de los otros ICEs. Así, mientras que los de las universidades presenciales españolas se concibieron como instrumentos ideales para impulsar y profundizar la reforma educativa promovida por la Ley General de Educación de 1970, el ICE de la UNED se concibió ya en 1972 como un instrumento para el conocimiento y desarrollo de la educación a distancia en la UNED.

El Rectorado de la Universidad le asignó entonces las funciones de: investigación institucional, formación del profesorado tanto de la Sede Central como de los Centros Asociados, determinación de los criterios metodológicos propios de esta modalidad educativa y aplicación de la tecnología educativa precisa.

El Claustro Constituyente de la UNED aprobó en sus Estatutos de 1985 una reforma consistente en la Transformación del ICE en Instituto Universitario de Educación a Distancia (IUED) con competencias en el ámbito de la metodología a distancia. Actualmente el IUED tiene encomendada la formación de los profesores de la Sede Central y de los profesores tutores en lo relacionado con la metodología de la enseñanza a distancia. Por otro lado es responsable de la investigación sobre la propia UNED, así como sobre la educación a distancia en general. El fruto de 
estas investigaciones nutre al resto de las actividades del Instituto, y suministra información relevante a la propia institución.

La evaluación institucional ha constituido otra de las competencias tradicionales del IUED y que actualmente encajan a la perfección en el Vicerrectorado de Innovación y Calidad creado durante el curso 2003-2004 y que ha pasado a denominarse Vicerrectorado de Calidad e Innovación Docente.

\section{El Centro de Diseño y Producción de Medios Audiovisuales (CEMAV).}

Por su propia denominación, en el CEMAV cabrían todos los medios audiovisuales: radio, video, videoconferencia, CD-ROM y televisión, pero se han dividido entre distintos departamentos o centros por razones de eficacia y por la complejidad y envergadura que ha ido tomando este ámbito de la enseñanza universitaria a distancia. Así, en este momento existen los servicios telemáticos, que se ocupan de la videoconferencia y el CD-ROM, la televisión educativa, con dirección autónoma, y el CEMAV, o Centro de Medios Audiovisuales, que se encarga de la radio universitaria y de la producción de audios y videos educativos.

El Departamento de Radio del CEMAV tiene la misión de realizar los programas radiofónicos, destinados, prioritariamente, a los alumnos de la UNED. Su función es llevar el control técnico y la organización de grabaciones y emisiones. En estos momentos se emiten diez horas semanales de radio a través de Radio Nacional de España. Este Departamento es también el encargado de la producción de audio que sirve de apoyo a las enseñanzas regladas y no regladas de nuestra Universidad.

El Departamento de Video del CEMAV tiene la misión de realizar, producir y editar los videos educativos, trabajando en equipo con los profesores interesados en este soporte didáctico. Actualmente, están en catálogo más de 150 videos, lo cual conforma una de las videotecas educativas más completas de España.

El Departamento de Televisión Educativa del CEMAV viene ofreciendo programas de televisión a través de la cadena pública de Televisión Española, La 2. Estos programas, siempre con nivel universitario, abarcan los grandes temas que pueden ser de interés para un público más allá de los propios estudiantes de la Universidad. 


\section{El Centro de Innovación y Desarrollo Tecnológico (CINDETEC).}

Este Centro nacido recientemente trata de dar respuesta a los siguientes retos esenciales:

- Mejorar el uso eficiente de las TIC en la UNED en todos los ámbitos: investigación, gestión y enseñanza/aprendizaje.

- Responder a la disposición adicional segunda de la LOU en la que se señala la "creación de un Centro Superior para la Enseñanza Virtual".

- Facilitar la colaboración, el desarrollo conjunto y la provisión de servicios TIC para otras entidades e instituciones.

- Garantizar la innovación continua en el uso de las TIC aplicadas a los procesos de enseñanza y aprendizaje, mediante sistemas centrados en las necesidades del usuario que consideren la accesibilidad como requisito básico, así como el desarrollo abierto y basado en estándares

\section{Innova: Sección de Innovación de la UNED}

Es objetivo principal de esta sección, el desarrollo de nuevas aplicaciones de enseñanza y aprendizaje, incluyendo nuevas versiones de las plataformas y servicios básicos utilizados. Además, está centrada en temas de investigación que permitan afrontar el futuro del uso adecuado de las TIC para potenciar los procesos de enseñanza y aprendizaje en una universidad de educación a distancia como la UNED. Desde el año 2000, Innova, en su labor de I+D dentro de la UNED, viene desarrollando la plataforma de e-learning aLF, con el fin de ofrecer un producto sólido, potente y ampliamente utilizado.

\section{LA EVALUACIÓN DE LOS APRENDIZAJES}

En la UNED, desde su creación, se ha sido consciente de que para muchos estudiantes va a ser la evaluación la que, casi exclusivamente, posibilite la relación profesor-estudiante. Es a través de ella cuando algunos docentes de la UNED han tenido la ocasión única de reorientar el aprendizaje de sus estudiantes, aunque sólo sea cuando éstos se ven obligados a cumplimentar las pruebas de evaluación a distancia o a realizar alguna prueba presencial. El modelo UNED prevé que los estudiantes puedan realizar tres tipos de pruebas, las de autoevaluación, las de evaluación a distancia y las presenciales (García Aretio, 1994; 1996). 


\section{Las pruebas de autoevaluación}

En los ejercicios de autoevaluación o autocomprobación que pueden integrarse en los materiales tanto impresos como electrónicos, se efectúan unas preguntas que el estudiante responde, bien eligiendo entre más de una opción, o elaborando la respuesta y, con inmediatez, puede comprobar el acierto o error de la misma, dado que las soluciones correctas las ofrece el autor del material, desde su propia perspectiva, en otro lugar distinto a aquel en el que aparecen las preguntas, estén éstas editadas en material impreso o electrónico.

\section{Las pruebas de evaluación a distancia}

Las pruebas de evaluación a distancia consisten en unos ejercicios en los que se plantean cuestiones a los estudiantes que éstos habrán de responder. Estas pruebas son estructuradas de muy diversas maneras, siempre en función de la naturaleza de la materia o curso que se imparte. Desde trabajos amplios que requieran poner en juego diversas capacidades cognitivas del estudiante que evalúan distintos objetivos de formación, hasta pruebas objetivas, pasando por pruebas de ensayo o trabajos de elaboración, sean éstos teóricos o prácticos. Estas son corregidas por el profesor tutor del Centro Asociado, que las devuelve al estudiante con las observaciones que crea oportunas.

Estas pruebas a distancia constituyen un medio fundamental de orientación en el estudio independiente. La modalidad, extensión y criterios de corrección de las pruebas de evaluación a distancia son establecidos libremente por el equipo docente de la asignatura, así como su formato, impreso o electrónico.

\section{Las pruebas presenciales}

Las pruebas presenciales son preceptivas en la UNED para todos los estudios reglados (de carácter formal) y son el medio fundamental de control del rendimiento académico de los estudiantes e instrumento primordial de calificación. La modalidad, características, extensión y organización internas de las pruebas son decididas por el equipo docente de cada asignatura, de acuerdo con los objetivos establecidos en su plan docente anual.

Estas pruebas presenciales se celebran en todos los Centros Asociados de España y del extranjero, en los meses de febrero, junio y septiembre de cada año. Estas pruebas ponen al estudiante en disposición de demostrar hasta qué punto los 
trabajos realizados a distancia han sido fruto de su exclusivo esfuerzo personal. En la mayoría de los cursos de enseñanzas no regladas no son obligatorias, sin embargo son numerosos los estudios de postgrado que exigen la realización de pruebas presenciales.

Antes de la corrección de las pruebas por parte de los profesores de la Sede Central, los profesores tutores de los Centros Asociados emiten un informe sobre cada uno de sus estudiantes en el que se hacen constar las calificaciones y valoración general de las pruebas y trabajos que hayan podido realizar los estudiantes, junto a aquellas otras observaciones que solicite el equipo docente o que el profesor tutor estime pertinentes. La calificación final del curso se otorga a la vista de las calificaciones de las pruebas presenciales, el informe tutorial y aquellos otros datos que el equipo considere necesarios.

Las calificaciones de cada una de las pruebas presenciales son comunicadas personalmente a los estudiantes a través de los sistemas tecnológicos de los que la UNED dispone, sistema automático de telefonía avanzado y, naturalmente, a través de Internet.

\section{PARA TERMINAR}

La UNED se configura hoy como la Universidad española con mayor número de estudiantes. Todas sus Facultades y Escuelas cuentan con el mayor número de estudiantes comparadas con sus homólogas de otras universidades presenciales de España.

La perspectiva internacional de la UNED es cada vez más prometedora, tanto por las posibilidades que ofrece a estudiantes residentes en el extranjero como por su dimensión y proyección en otros países, fundamentalmente en Iberoamérica, donde cuenta con gran número de alumnos, sobre todo en los programas de postgrado. $\mathrm{Su}$ pertenencia a la European Association of Distance Teaching Universities (EADTU) que es la más representativa organización europea en el ámbito de la educación a distancia universitaria y a la homóloga Asociación Iberoamericana de Educación Superior a Distancia (AIESAD), atestiguan el interés por esta dimensión internacional.

En otro orden de cosas, la investigación cubre buena parte de la dedicación del profesorado de esta Universidad. Sus anuarios de investigación recogen todo lo que cada año se produce en el ámbito científico. 
Y, finalmente, la UNED va profundizando en la cultura interna de la calidad de sus titulaciones, programas, gestión, servicios, etc. La preocupación por la calidad ocupa hoy a la totalidad de las personas que laboran en esta Universidad. Un Vicerrectorado dedica todos sus esfuerzos a la mejora de la calidad y a su evaluación.

\section{DOCUMENTOS LEGALES RELATIVOS A LA CREACIÓN Y DESARROLLO DE LA UNED}

- Ley General de Educación (LGE) 14/1970 de 4 de agosto. (Boletín Oficial del Estado de 6 de agosto de 1970).

- Decreto 1106/1971 de 6 de mayo que crea la Comisión Gestora para el establecimiento de la modalidad de enseñanza universitaria a distancia. (Boletín Oficial del Estado de 1 de junio de 1971).

- La Ley 22/72 de 10 de mayo por la que se aprueba el III Plan de Desarrollo Económico y Social para el cuatrieno 72/75, autoriza al Gobierno para crear dentro de ese cuatrienio, entre otras, la Universidad Nacional de Educación a Distancia. (Boletín Oficial del Estado de 20 de mayo de 1972).

- Decreto 2310/1972 de 18 de agosto por el que se creó la Universidad Nacional de Educación a Distancia (UNED). (Boletín Oficial del Estado de 9 de septiembre de 1972).

- Decreto, el 3114/1974 de 25 de octubre se establece el marco legal mediante el que ha venido funcionando la Universidad hasta la aprobación de sus Estatutos e incluso hasta nuestros días. (Boletín Oficial del Estado de 14 de noviembre de 1974).

- Estatutos de la Universidad Nacional de Educación a Distancia. Real Decreto 1287/1985, de 26 de junio (BOE de 31 de julio) y complementados por el texto aprobado por Real Decreto 594/1986, de 21 de febrero (Boletín Oficial del Estado de 28 de marzo de 1986).

- Nuevos Estatutos de la Universidad Nacional de Educación a Distancia. Real Decreto 426/2005, de 15 de abril de 2005 (Boletín Oficial del Estado de 16 de abril de 2005).

- Ley Orgánica de Reforma Universitaria de 25 de agosto de 1983 (Boletín Oficial del Estado de 1 de septiembre de 1983).

- Real Decreto 1317/1995, de 21 de julio por el que se crea la Red Básica de Centros Asociados y perfila el régimen de convenios. (Boletín Oficial del Estado de 10 de agosto de 1995).

- Ley Orgánica de Universidades (LOU) 6/2001 de 21 de diciembre (Boletín Oficial del Estado de 24 de 24 de diciembre de 2001). 
- Reforma de la Ley Orgánica de Universidades (LOU), 2007, aprobada por las Cortes Generales y Senado.

\section{NOTAS}

1 En la presente tabla también se han contabilizado los alumnos de los planes a extinguir sumándolos a los correspondientes de las titulaciones actuales que sustituyen a las anteriores.

2 Aunque en breve se establecerán los créditos europeos ECTS, todavía en las universidades españolas un crédito equivale a 10 horas de docencia. El ECTS equivaldrá a 25-30 horas de trabajo del estudiante.

\section{REFERENCIAS BIBLIOGRÁFICAS}

García Aretio, L. (1985). Licenciados extremeños de la UNED. Mérida. Badajoz: UNED-Mérida.

García Aretio, L. (1986). Educación superior a distancia. Análisis de su eficacia. Badajoz: UNED-Mérida.

García Aretio, L. (1987). Rendimiento académico y abandono en la educación superior a distancia. Madrid: ICEUNED.

García Aretio, L. (1994). Educación a distancia hoy. Madrid: UNED.

García Aretio, L. (1995). "Decisiones políticas para la creación y consolidación de una universidad singular: la UNED”. $V$ Congreso Interuniversitario de Teoría de la Educación "Política y educación". Barcelona: Universidad Autónoma, pp. 373-380.

García Aretio, L. (1995). "Advantages and disadvantages of a institutions: Spain's UNED”. En Sewart, D. (Ed.) One World Many Voices. Quality in Open and Distance Learning. Milton Keynes: The Open University-ICDE, pp. 92-95.

García Aretio, L. (1996). La educación a distancia y la UNED. Madrid: UNED.
García Aretio, L. (Coord.) (1997). Unidades didácticas y guías didácticas. Orientaciones para su elaboración. Madrid: UNED.

García Aretio, L. y otros (1997). Plan para la mejora de la calidad de la UNED. Madrid: UNED.

García Aretio, L. (1998). Universidad Nacional de Educación a Distancia. En: Vázquez, G. Madrid: espacio universitario abierto. Madrid: Fundación UniversidadEmpresa, pp. 261-300.

García Aretio, L. (1999). Historia de la educación a distancia. Revista Iberoamericana de Educación a Distancia (RIED). 2,1, pp. 11-40.

García Aretio, L. y otros (1999). ¿Qué piensan los estudiantes sobre su Universidad? Madrid: UNED.

García Aretio, L. (2001). La educación a distancia. De la teoría a la práctica. Barcelona: Ariel.

García Aretio, L. (2001). The Technological Consolidation of UNED in Spain. International Review of Research in Open and Distance Learning. 2, 1.

García Aretio, L. (2002). Distance Education 
Open University (Universidad Nacional de Educación a Distancia - UNED). En Venugopal, R. y Manjulika, S. Towards Virtualization. Open and Distance Learning. New Delhi: Kogan Page, pp. 556-595.

Ministerio de Educación y Ciencia (1995). La educación abierta y a distancia en España. Madrid: MEC/ Unión Europea. Sánchez Elvira, A. (2003). Informe de la investigación: Perfiles Psicosociales y rendimiento académico de los alumnos de la UNED. Disponible en: http:// portal.uned.es/pls/portal/docs/PAGE/ UNED MAIN/LAUNIVERSIDAD/ VICERRECTORADOS/CALIDAD E INNOVACION/INNOVACION DOCENTE/IUED/INSTITUCIONAL/ BLOQUE\%2OSOCIODEMOGR\%C $3 \% 81$ FICO\%2OINFORME\%20PERFILES\%2 oY\%20RENDIMIENTO.PDF [Consulta, 2007, 12 enero]

UNED (2006). Anuario estadístico de la UNED. Madrid: UNED-IUED.

\section{PERFIL ACADÉMICO Y PROFESIONAL DEL AUTOR:}

Lorenzo García Aretio. Decano de la Facultad de Educación de la UNED (www. uned.es/educacion), Titular de la Cátedra UNESCO de Educación a Distancia (www. uned.es/cued), Editor del Boletín Electrónico de Noticias de Educación a Distancia (www.uned.es/bened). Participa en numerosos proyectos europeos de investigación. Director de la revista científica Educación XX1. Más datos sobre su perfil, http:// www.uned.es/cued/curriculum.htm

DIRECCIÓN DEL AUTOR

Lorenzo García Aretio

Facultad de Educación - UNED

Paseo Senda del Rey, 7

28040 - Madrid, España

Fecha de recepción del artículo: 01/02/07

Fecha de aceptación del artículo: 15/03/07 